\title{
Philonsorbonne
}

8 | 2014

Année 2013-2014

\section{Le problème de la « sphère publique » dans la pensée de Carl Schmitt}

\section{Tommaso VISONE}

\section{(2) OpenEdition}

Journals

Édition électronique

URL : https://journals.openedition.org/philonsorbonne/626

DOI : 10.4000/philonsorbonne.626

ISSN : 2270-7336

Éditeur

Publications de la Sorbonne

\section{Édition imprimée}

Date de publication : 1 janvier 2014

Pagination : 101-210

ISSN : 1255-183X

\section{Référence électronique}

Tommaso VISONE, «Le problème de la « sphère publique » dans la pensée de Carl Schmitt »,

Philonsorbonne [En ligne], 8 | 2014, mis en ligne le 19 janvier 2014, consulté le 08 juin 2021. URL:

http://journals.openedition.org/philonsorbonne/626; DOI : https://doi.org/10.4000/philonsorbonne. 626

(c) Tous droits réservés 


\title{
Le problème de la «sphère publique " dans la pensée de Carl Schmitt
}

\author{
Tommaso VISONE
}

Quand on parle de la sphère publique, il n'est pas habituel d'entendre le nom de Carl Schmitt. En fait le juriste de Plettenberg n'a jamais été considéré comme l'un des théoriciens allemands de la Öffentlichkeit, titre qui a notamment été attribué à des penseurs comme Jürgen Habermas, Hannah Arendt, etc ${ }^{1}$. Mais toute la pensée de Carl Schmitt a été traversée par l'élaboration et l'utilisation constante des catégories de «privé » et de « public $»^{2}$. Serait-il donc possible d'identifier une conception spécifique

1. Voir les deux ouvres allemandes les plus étudiées à ce sujet: H. Arendt, Condition de l'homme moderne, Paris, Calmann-Lévy, 1983 et J. Habermas, L'Espace public : archéologie de la publicité comme dimension constitutive de la société bourgeoise, Paris, Payot, 1997. Pour la critique habermassienne_de la conception de la sphère publique chez Schmitt, on peut voir J. Seitzer et C. Thornhill, «An Introduction to Carl Schmitt's Constitutional Theory : Issues and Context », in C. Schmitt, Consitutional Theory, Durham, Duke University Press, 2008, p. 37-42.

2. Aujourd'hui la littérature critique sur la pensée de Schmitt est très vaste et ne peut être citée exhaustivement dans cet article. Il faut rappeler, pour le débat français récent, J.-F. Kervégan, Que faire de Carl Schmitt?, Paris, Gallimard, 2011; T. Storme, Carl Schmitt et le marcionisme, L'impossibilité théologico-politique d'un œecuménisme judéo-chrétien ?, Paris, Cerf, 2008 ; A. de Benoist, Carl Schmitt actuel. "Guerre juste », terrorisme, état d'urgence, "Nomos de la terre», Paris, Krisis, 2007 ; Y.-C. Zarka, Un détail nazi dans la pensée de Carl Schmitt, Paris, PUF, 2005 et le travail - effectué sous la direction d'A. de Benoist des éditions Krisis qui ont publié des ouvrages de Schmitt avec des essais critiques de G. Maschke et D. Zolo (Machiavel-Clausewitz. Droit et politique face aux défis de l'histoire, Paris, Krisis, 2007 et Guerre discriminatoire et logique des grands espaces, Paris, Krisis, 2011). Pour le débat à l'extérieur de la France, il suffit de signaler ici des textes célèbres comme C. Galli, Genealogia della politica. Carl Schmitt e la crisi del pensiero politico moderno, Bologne, Il Mulino, 1996 ; Id., Lo sguardo di Giano. Saggi su Carl Schmitt, Bologne, Il Mulino, 2008 ; J.-W. Müller, A Dangerous Mind. Carl Schmitt in Post-War European Thought, New Haven-Londres, Yale University Press, 2003 ; P. Tommissen, In 
de la «sphère publique» chez Schmitt? Et quand le juriste allemand parle de "sphère publique », s'agit-il de la même chose que lorsqu'on parle « d'espace public»?

D'abord, pour mieux comprendre ce problème, il faut, en fait, distinguer entre «sphère» (Sphäre), comme dimension de l'action et de la vie humaine, et «espace» (Raum) comme définition d'une zone, d'un lieu délimité où se joue la relation entre une idée politique et un territoire ${ }^{3}$. Donc, si pour Carl Schmitt il y a toujours eu une distinction entre «sphère publique » et «sphère privée », cela ne coïncidait pas avec l'« espace » au sens où nous venons de le définir. Dans ce sens, il ne peut y avoir d' «espace privé » (ni donc d' «espace public »). Il faut par conséquent se concentrer sur la dichotomie public/privé du point de vue de la « sphère ».

$* * *$

1. Schmitt s'est toujours confronté à des logiques dichotomiques (guerre/guerre civile; ami/ennemi ; Léviathan/Béhémot, etc.). Sa thèse de maîtrise en droit - Über Schuld und Schuldarten. Eine terminologische Untersuchung (1910) - est entièrement consacrée à la distinction entre sphère intérieure (du péché) et sphère extérieure (de la culpabilité), et à l'intérêt que le droit doit porter à la sphère intérieure en cas de dol et de négligence, comme moment où la sphère intérieure prend une grande importance pour la sphère extérieure ${ }^{4}$. Il faut également souligner comment Schmitt a toujours pensé la dichotomie public/privé à partir d'un problème de relation dans la distinction, comme il l'avait fait avec la distinction entre sphère intérieure et extérieure. À partir de cette idée complexe, Schmitt va aussi élaborer progressivement sa définition du «public» (öffentlich) et de la «sphère publique » (Öffentlichkeit) dans une logique dialectique avec le «privé» (privat) et la «sphère privée» (Privatsphäre). On peut dire « progressivement », parce qu'il est difficile de trouver une définition directe du «public» dans toute l'œuvre de Schmitt, tandis qu'une caractérisation constante de la dichotomie public/privé se dessine peu à peu dans les écrits schmittiens jusqu'à la deuxième guerre mondiale. Â partir des années 1920 tout particulièrement, cette caractérisation s'enrichit de l'analyse que Schmitt consacre à l'État moderne et à sa crise, qu'il voyait se manifester dans les difficultés rencontrées par la République de Weimar. C'est donc

Sachen Carl Schmitt, Vienne, Karolingen, 1997 et les «Schmittiana», sous la direction du même Tommissen, huit volumes différents parus entre 1988 et 2003 en diverses éditions. Très intéressant aussi, le récent volume Thomas Hobbes and Carl Schmitt. The politics of order and myth, sous la direction de J. Tralau, Londres, Routledge, 2012.

3. Voir C. Schmitt, Le Droit des peuples réglé selon le grand espace proscrivant l'intervention de puissances extérieures. Une contribution au concept d'empire en droit international (1939-1942), in C. Schmitt, Guerre discriminatoire, op . cit., p.159.

4. Voir C. Schmitt, Über Schuld und Schuldarten. Eine terminologische Untersuchung, Breslau, Schletter, 1910. Voir aussi sur ce point S. Pietropaoli, Schmitt, Rome, Carocci, 2012, p. $18-19$. 
principalement en relation à l'âge moderne que Schmitt parle du «public » et du « privé » 5 .

2. Où sont les caractéristiques attachées à cette opposition, et quelles sont-elles? Dans l'œuvre Politische Romantik (1919) Schmitt analyse l' «occasionnalisme subjectivisé » qui est typique, selon le juriste, du romantisme ainsi que de l'Allemagne d'après-guerre. Le romantisme voit la naissance d'un mysticisme individualiste qui fait de l'individu, seul et isolé, le centre d'un monde solipsiste. Ce mouvement de la pensée est, selon Schmitt, un prélude au primat de l'économie et à la privatisation de la vie qu' effectuera ensuite le libéralisme ${ }^{6}$. Ainsi le romantique, qui est un citoyen bourgeois "privé », n'est jamais politique, parce qu'il ne décide (entscheiden) jamais et se distingue aussi du politique romantique (Don Quijote), qui est lui capable de décision et d'action publique ${ }^{7}$. On trouve donc ici une première esquisse d'identification entre ce qui est individuel, solipsiste, irresponsable et privé, en opposition à une responsabilité publique et politique.

En 1923, avec l'œuvre Die geistesgeschichtliche Lage des heutigen Parlamentarismus la caractérisation qu'opère Schmitt se précise et s'enrichit du point de vue historique. L'écrit est consacré à une critique radicale du libéralisme et de son expression institutionnelle, le parlementarisme. Schmitt affirme ici que l'idée libérale du «public », liée à la conviction de pouvoir obtenir une «opinion publique » grâce à la «publicité » de l'opinion de l'individu, est fausse et dangereuse. En fait, pour le juriste allemand, le droit de manifestation de l'opinion individuelle est seulement un moyen d'introduire le «privé » dans le « public ». Il écrit :

Où la publicité peut devenir constriction, comme dans l'exercice du droit de vote par les individus, ici dans le point de passage du privé au public succède, donc, l'exigence antithétique au secret électoral. La liberté d'opinion est une liberté des personnes privées ${ }^{8} \ldots$

La conséquence est que, avec l'entrée des intérêts et des opinions privés dans la vie publique - phénomène que la politique de masse ne fait que renforcer - le libéralisme a fini par masquer sa logique propre en créant une

\footnotetext{
5. En effet, Schmitt «privilégiait » l'État moderne comme terrain d'enquête historique. Voir G. Miglio, Presentazione, in C. Schmitt, Le categorie del politico, Bologne, Il Mulino, 1972, p. 8-9.

6. Voir C. Schmitt, Romanticismo politico, Turin, Giuffrè, 1981, p. 233-239. Sur le rapport entre romantisme et libéralisme voir aussi C.Schmitt, La condizione storico-spirituale dell'odierno parlamentarismo, Turin, Giappichelli, 2004, p. 50-51.

7. Comme le montre l'exemple de Sand, voir C. Schmitt, Romanticismo politico, op. cit., p. 213-215.

8. C. Schmitt, La condizione storico-spirituale, op. cit., p. 55
} 
publicité de «façade », tout en opérant un retour au secret dans les prises de décision les plus importantes pour l'intérêt public. Il écrit :

De petites et minuscules commissions de partis et coalitions de partis décident derrière des portes cloisonnées, et tout ce que les représentants du grand capitalisme concluent est, peut-être, encore plus important que les décisions «politiques» pour la vie quotidienne et pour le destin des millions d'hommes'.

Schmitt montre ici comment le secret du pouvoir devient le secret du privé, assuré par les droits et les idées du libéralisme. Pour Schmitt, le libéralisme garantit ainsi l'invasion du public par le privé au XIX ${ }^{\mathrm{e}}$ siècle ${ }^{10}$. C'est une thèse qu'il étaiera avec beaucoup plus d'arguments en 1938 dans son étude sur Hobbes, mais elle est introduite dans ses éléments essentiels en 1923. Il est possible de voir aussi comment Schmitt avance une association entre économique/privé et politique/public, où l'élément économique - et particulier de la politique (qui n'est pas le politique pour Schmitt) - va gagner progressivement sur le politique ${ }^{11}$.

\section{*** $*$}

3. Mais la dichotomie va s'enrichir encore plus avec le temps. En 1927, dans le concept du politique (Der Begriff des Politischen) Schmitt met en évidence comment :

Les concepts d'ami et d'ennemi doivent être pris dans leur signification concrète, existentielle, pas comme métaphores ou symboles ; ils ne doivent pas être mélangés et affaiblis par des conceptions économiques et morales ou d'un autre type et, encore moins, doivent-ils être entendus au sens individualisteprivé, comme expression psychologique des sentiments et des tendances privés... L'ennemi n'est pas le concurrent ou l'adversaire en général. L'ennemi n'est pas non plus l'adversaire privé qui nous hait selon des sentiments d'antipathie. L'ennemi est seulement un ensemble d'hommes qui combattent au moins virtuellement, c'est-à-dire selon une possibilité réelle, et qui s'opposent à un autre ensemble humain du même type. L'ennemi est seulement l'ennemi public, parce que tout ce qui se réfère à un tel ensemble, et en particulier à un peuple entier, devient pour cette même raison public ${ }^{12}$.

Ici le public est tout ce qui regarde un ensemble d'hommes - et en particulier un peuple - qui est en condition de combattre, y compris

\section{Ibid., p. 71.}

10. C'est une thèse qui trouvera son principal développement dans La notion du politique (1927-1932). Voir C. Schmitt, Il concetto di politico, in Id., Le categorie del politico, op. cit., p. 158.

11. En particulier grâce à la relation entre les partis et le capital. Ibid., p. 25-29.

12. C. Schmitt, Il concetto di politico, in Id., Le categorie del politico, op. cit., p.110-111. 
virtuellement $^{13}$. C'est par conséquent un concept qui concerne une collectivité et pas une individualité, surtout quand cette collectivité est un peuple. En effet, comme le dit Gwénaël Le Brazidec, Carl Schmitt insiste beaucoup sur la relation entre public et peuple :

Pour lui la politique appartient obligatoirement à la sphère publique ainsi qu'il l'explique en prenant l'exemple du couple ami/ennemi : l'ennemi n'est pas l'inimicus, celui avec lequel on n'est pas ami à titre privé mais l'hostis, c'est-à-dire l'ennemi public. La démocratie étant une forme politique au sens schmittien, elle appartient obligatoirement à l'espace public ce qui amène Carl Schmitt à donner sa définition de l'expression de la volonté populaire qui ne peut manquer de surprendre au regard des principes de la démocratie libérale... Fondant la démocratie sur l'identité entre la volonté populaire et la loi, Schmitt souligne les implications de la notion de peuple après avoir rappelé que celle-ci est souvent définie négativement, par rapport au système politique dominant, comme une masse non distinguée. Mais cette notion de peuple n'existe que dans l'espace public; seul le peuple présent, physiquement rassemblé, est peuple et constitue la vie publique. C'est sur ce fondement que, selon Schmitt, repose l'idée de Rousseau selon laquelle le peuple ne peut être représenté : il doit être présent s'il veut exister. Les exemples de véritable démocratie sont donc, pour Schmitt, l'Agora, le Forum ou bien encore les Assemblées cantonales helvétiques... ${ }^{14}$

Le problème du régime représentatif et de ses textes constitutionnels est d'ignorer la dimension publique, où se manifeste le souverain: «les véritables assemblées populaires et les acclamations ». Les formes modernes de manifestation de la souveraineté sont le référendum et l'initiative populaire parce que, en ouvrant un vrai débat public entre les citoyens, ils finissent par être expressions du public et pas du privé ${ }^{15}$. Au contraire, la démocratie représentative construite par les libéraux n'est qu'une fausse démocratie qui organise la discussion publique uniquement autour d'une

13. On pourrait soutenir que, pour avoir un «ennemi », il faut avoir un ensemble d'hommes en condition de frapper un autre ensemble. On peut donc se demander: est-ce que la vie collective, populaire, et donc le «public » sont, pour Schmitt, une condition de l' « ennemi » et donc du «politique»? Sans peuple, groupe menacé/menaçant, il n'y a pas un hostis, seulement un inimicus; s'agit-il là d'une condition logique du politique ? Ce point est important pour essayer de comprendre la relation entre décisionnisme et institutionnalisme (ordre concret) dans la pensée de Schmitt.

14. G. Le Brazidec, René Capitant et Carl Schmitt : crise et réforme du parlementarisme. De Weimar à la Ve République, Paris, L'Harmattan, 1998, p. 194-195.

15. En 1927 - dans Volksentscheid und Volksbegehren - il insiste sur l'identité entre privé / irresponsabilité et public/ responsabilité en mettant en évidence comment le vote secret individuel, prévu par le système représentatif, n'est qu'une modalité pour transformer le votant en un «privé » et donc en un «irresponsable» et en un «solitaire ». Dans le système représentatif, le peuple n'est que le résultat d'une addition entre des particularités (c'est Rousseau qui inspire Schmitt ici). Voir C. Schmitt, Referendum e proposta di legge d'iniziativa popolare. Un contributo all'interpretazione della costituzione weimariana ed alla dottrina della democrazia diretta, in C. Schmitt, Democrazia e liberalismo, Milan, Giuffrè, 2001, p. 62-67. 
propagande et d'un système de presse dominés par des instances privées. D'où la tendance à identifier la volonté publique comme l'addition de volontés privées (individuelles, secrètes et isolées), et l'affirmation de la technique comme instrument orienté vers la dépolitisation définitive de la société. Voilà la prophétie du juriste préoccupé par cette alliance entre privé et technique :

On pourrait imaginer qu'un jour de subtiles inventions permettraient à chacun d'exprimer à tout moment ses opinions sur les problèmes politiques sans quitter son domicile, grâce à un appareillage qui ferait enregistrer automatiquement toutes ces opinions sur une centrale où on n'aurait plus qu'à lire le résultat ${ }^{16}$.

Tout cela serait, pour lui, une dangereuse mise en discussion du «public » et, avec lui, du peuple et de la démocratie ${ }^{17}$. Le libéralisme, donc, en travaillant pour le privé (pour l'isolement contre la discussion, pour l'individu contre le peuple, pour la société contre l'État) est en train de tuer la démocratie. Cette vision sera, encore une fois, reprise dans Le gardien de la Constitution (Der Hüter der Verfassung, 1931) ${ }^{18}$ et dans Légalité et Légitimité (Legalität un Legimität, 1932) ${ }^{19}$ qui ont étés écrits au temps du crépuscule de Weimar. Durant cette période Schmitt élabore aussi son concept d' «État total par faiblesse » ou d' «État quantitatif ${ }^{20}$ qui est un État qui augmente son volume sans grandir en intensité et énergie politique. Un État où l'élément «public » est prisonnier du «privé », des intérêts particuliers de la société (partis, capitalistes, syndicats, etc.). Contre cet État, Schmitt, au début des années 1930, pense qu'il est encore possible d'agir en introduisant dans le système de Weimar une neutralisation active, ou politique/publique : un État total par intensité, ou qualitatif, qui aurait eu le gardien de la constitution (le président du Reich) comme sommet politique ${ }^{21}$.

$* * *$

4. Jusqu'ici on a vu comment Schmitt conçoit la sphère publique comme la sphère de la collectivité, de l'État, du Visible, de la manifestation du

16. C. Schmitt, cité dans G. Le Brazidec, op. cit., p. 196.

17. Cette question est aussi traitée dans la Verfassungslehre en 1928. Voir C. Schmitt, Constitutional Theory, op. cit., p. 274-277.

18. Voir C. Schmitt, Der Hüter der Verfassung, Tübingen, Mohr, 1931.

19. Voir C. Schmitt, Legalität und Legimität, Monaco-Lipsia, Duncker \& Humblot, 1932.

20. Voir C. Schmitt, «Starker Staat und gesunde Wirtschaft», Mitteilungen des Vereins zur Wahrung der gemeinsamen wirtschaftlichen Interessen in Rheinland und Westfallen, $\mathrm{n}^{\circ} 1$, Düsseldorf, Strucken, 1932, p. 13-32.

21. Voir C. Schmitt, Sviluppo ulteriore dello Stato totale in Germania, (1933), in Id., Posizioni e Concetti in lotta con Weimar-Ginevra-Versailles 1923-1939, Milan, Giuffrè, 2007, p. 305-307. 
peuple, de la responsabilité supra-individuelle, de la discussion, du sens ${ }^{22}$, et de la politique («le politique » ayant toujours un lien très fort avec le public) contre un privé qui est vu comme la sphère de l'individualité, de la société, du secret (de l'invisible), de la subjectivité solipsiste, de l'isolement, du non-sens, de l'irresponsabilité et de l'économie. En même temps on a vu que Schmitt pense ces deux sphères en relation dynamique l'une avec l'autre et comment il voit, dans le libéralisme, l'idéologie et la métaphysique qui amènent la sphère privée à s'imposer progressivement sur la sphère publique, jusqu'à abolir la distinction entre les deux (État total par faiblesse) et, en définitive, à détruire l'État. Toute sa vision du problème public/privé va évoluer pendant les années 1930. En fait, après 1933, Schmitt assiste à la fin de la République de Weimar et, avec elle, à l'émergence du nazisme. Le second lui semble être l'idéologie adéquate pour réaliser sa nouvelle idée de l' «Ordre Concret»(Konkrete Ordnung) $)^{23}$ et il commence donc à reconsidérer le problème à travers le prisme de ce nouveau régime. En 1933, dans Staat, Bewegung, Volk: Die Dreigliederung der politischen Einheit, Schmitt affirme que le nouveau système nazi (composé de l'État, du mouvement et du peuple) est capable d'aller au-delà de la séparation entre public et privé, parce que c'est une structure politique nouvelle et non un État classique. Par conséquent, la conception nazie de l'État tripartite est supérieure à la structure à deux parties du vieil État (État/société, Droit/État, public/privé, etc.), parce qu'il est capable, contre la dépolitisation, d'instituer et de rendre effectif le primat du «politique » sur l' «apolitique », grâce à l'action dynamique du mouvement/parti qui véhicule une idée commune dans tout le corps du système, concrètement unifié dans le partage concret du sang $^{24}$. La décision politique, ainsi, va imposer sa capacité de transformation à tous, sans possibilité de se «soustraire à la collaboration » $(1936)^{25}$. Tous les problèmes du vieux dualisme vont disparaître dans la nouvelle construction du nazisme qui s'oppose au vrai danger de l'époque de la

22. Schmitt est ici hégélien en identifiant le public/état avec la construction d'une sphère de sens collective qui unifie la société (ce qui pour Hegel est la Sittlichkeit, l'éthicité) ; une chose que le repli bourgeois des romantiques dans le privé rend impossible. Sur la relation entre la pensée de Hegel et celle de Schmitt, voir aussi J.-F. Kervégan, Hegel, Carl Schmitt. La politique entre spéculation et positivité, Paris, PUF, 2005.

23. Comme le dit S. Pietropaoli, selon la théorie de l'ordre concret, la décision est prise à l'intérieur des institutions et des concepts juridiques typiques d'un peuple et de son histoire, liée à une particularité qui en exclut toute universalisation et formalisation. Voir S. Pietropaoli, Schmitt, op. cit., p. 109-115, et Id., « Il diritto internazionale come ordinamento concreto », in A. Buela, D. Cumin \& S. Pietropaoli, Carl Schmitt : Diritto e concretezza, Parme, Edizioni all'insegna del Veltro, 2011, p. 68-74. Sur le «virage» institutionnaliste de Schmitt pendant les années 1930, voir C. Schmitt, Les trois types de pensée juridique, (1934), Paris, PUF, 1995.

24. Voir C. Schmitt, Stato, Movimento, Popolo, (1933), in Id., I principi politici del nazionalsocialismo. Scritti scelti e tradotti da Delio Cantimori, Florence, Edizioni Movimento, 1935, p. 175-176.

25. Pour Schmitt, en fait, on est dans une « époque intégralement politique de la civilisation ». Id., L'Era della politica integrale, (1936), in C. Schmitt, L'Unità del mondo e altri saggi, sous la direction d'A. Campi, Rome, Pellicani Editore, 1994, p. 87-89. 
« fin » $\left(\right.$ Auflösung $\left.{ }^{26}\right)$ de l'État : l'ouverture d'une guerre civile transnationale alimentée par le libéralisme et par l'universalisme ${ }^{27}$. Un danger auquel le régime allemand s'oppose en affirmant une alternative. Cette préoccupation est directement abordée en 1938 dans deux écrits: Die Wendung zum diskriminierenden Kriegsbegriff et Der Leviathan in der Staatslehre des Thomas Hobbes: Sinn und Fehlschlag eines politischen Symbols. Dans le deuxième - consacré à la destruction de l'État par l'intérieur - Schmitt identifie la raison de la crise de l'État (de la mort du Léviathan) à l'introduction hobbesienne d'une distinction rigide entre public et privé. Elle est liée au problème de la relation entre miracle et foi :

Ici où on parle du miracle et de la foi, Hobbes touche le point décisif... $\mathrm{C}^{\prime}$ est le lieu où la distinction entre foi intérieure et confession extérieure fait irruption dans le système politique du Léviathan. Hobbes considère la question des prodiges et des miracles comme une affaire de la raison publique en opposition à la raison privée, mais, en raison d'une liberté générale de pensée quia cogitatio omnis libera est - laisse à l'individu la liberté intérieure de croire ou pas - selon lui-même et selon sa raison privée - et de garder son «judicium» particulier en son cœur «intra pectus suum »... Les distinctions de public et privé, foi et confession, sont ainsi introduites d'une façon telle que chaque développement ultérieur, pendant le siècle suivant jusqu'au libéral Etat constitutionnel de droit, en découle en conséquence... Au moment où la distinction entre intérieur et extérieur est reconnue, la supériorité de l'intérieur sur l'extérieur, et donc du privé sur le public, est, en substance, une chose déjà décidée. Un pouvoir public pour autant qu'il soit loyalement respecté, comme pouvoir seulement public et seulement extérieur est vide et sans âme à l'intérieur... Qui s'est fait toucher par l'opposition entre intérieur et extérieur, en général a déjà reconnu par là, à la fin, la supériorité de l'intérieur sur l'extérieur, de l'invisible sur le visible, du silence sur la parole, de l'au-delà sur l'ici bas... Le Léviathan comme "magnus homo", comme souveraine personnification de l'État en forme divine, a été détruit de l'intérieur... Pour le Dieu mortel la distinction entre intérieur et extérieur a été la maladie qui l'a conduit à la mort ${ }^{28}$.

C'est donc la réserve d'un espace privé de la conscience, posée par Hobbes (et, avec une importante inversion d'accent, par Spinoza), qui donne la raison de la future destruction de l'État, identifiée avec l'extériorisation du public (qui ne peut pas connaître ni s'occuper de la «vérité substantielle») et la progressive avancée du privé qui, à la fin, tue le «magnus homo». Schmitt donne ici sa dernière caractérisation importante du public - comme

26. Dissoudre, glisser progressivement dans le point de chaos. Sur ce point voir S. Pietropaoli, Nota al testo, in C. Schmitt, Il concetto discriminatorio di guerra, Rome, Laterza, 2008, p. XL.

27. On peut voir à ce propos T. Visone, «Réflexions sur la guerre civile à l'époque des idéologies (1929-1939). Carl Schmitt et Karl Polanyi », Giornale di Storia Costituzionale, $\mathrm{n}^{\circ} 26,2 / 2013$ (en cours de parution).

28. C. Schmitt, Sul Leviatano, Bologne, Il Mulino, 2012, p. 93-103. 
extérieur, visible, parole, ici-bas - et du privé - comme intérieur, invisible, silence, au-delà. Après l'écrit sur Hobbes, le juriste de Plettenberg ne reviendra plus sur les problèmes intérieurs de l'État moderne et se consacrera aux grandes questions du droit international ${ }^{29}$.

$* * *$

5. Pour conclure cet exposé, on peut souligner la façon dont Schmitt, avec son étude du rapport public-privé, montre qu'il n'est pas possible de séparer une sphère de la vie humaine de l'autre sans aboutir à une fin des sphères tout court, dans la mesure où la sphère protégée - la sphère privée agresse l'autre. Il nous donne toujours une acception évaluative, et non simplement descriptive, des deux sphères; elle est caractérisée par la capacité de la sphère privée à réussir à créer, avec succès, une dimension protégée, là où elle peut cultiver la plus agressive des armes : la Vérité. Pour cette raison la sphère privée est destinée, à l'âge moderne, à provoquer la fin du Léviathan et de la dichotomie public/privé. La description par Schmitt du régime de Weimar, de son écroulement, nous montre comment en Allemagne, à la fin des années 1920, il n'y avait plus de sphère publique mais plus non plus de sphère privée clairement délimitée. De ce point de vue, le choix du nazisme ne signifie pas que jusqu'en 1933 Schmitt était favorable à la suppression de la dichotomie, mais simplement qu'il pensait le rapport entre les deux sphères - distinctes mais pas séparées - comme une société par actions où le golden share de la Vérité était propre au public, autrement dit comme une dynamique où le moteur de la légitimité et de la création du sens était le public et pas le privé, le politique et pas l'«économique ». Cela afin de garantir l'unité politique - de l'État - qui aurait éloigné le spectre d'une guerre civile toujours possible. Le choix du nazisme en 1933 sera en continuité avec cette exigence. Le temps de l'État moderne s'étant clos avec la chute de Weimar, il fallait une alternative « concrète » à la crise de l'État - c'est la période du Konkrete Ordnung, de l'hybridation entre décisionnisme et institutionnalisme - et le Reich allemand, avec sa nouvelle idéologie fondée sur l'élément concret du sang, semblait une réponse. Une alternative à laquelle Schmitt aurait travaillé jusqu'en $1940^{30}$. Après cela, le «Benito Cereno ${ }^{31}$ » aurait davantage travaillé sur l'exigence d'un nouveau nomos que sur la caractérisation et l'amélioration de l'ancien. Aujourd'hui on peut penser que les problèmes

29. Il y a, bien sûr, dans le «Nomos de la terre » des références au public et au privé, mais elles sont historiques (histoire du territoire et de la propriété) et confinées en peu de lignes. Le même discours est valide pour le Glossarium (où, en 1947, il dit être en accord avec Hariou pour considérer le «territoire» comme «public»). Voir C. Schmitt, Glossario, Milan, Giuffrè, 2001, p. 18.

30. En 1940 il écrivait «Reich und Raum. Elemente eines neuen Volkerrechts » (Zeitschrift der Akademie für Deutschland Recht 7, $\left.\mathrm{n}^{\circ} 13\right)$.

31. C'est le nom du protagoniste d'un roman de Hermann Melville que Schmitt utilise pour décrire sa condition après la deuxième guerre mondiale. Voir C. Schmitt, Ex captivitate salus, textes présentés par A. Dorémus, Paris,Vrin, 2003. 
soulignés par cette figure discutable ne doivent pas être sic et simpliciter assimilés aux choix politiques de son auteur (qui sont bien sûr liés de manière non négligeable à sa pensée). La réflexion sur la question de l'État et donc sur la sphère publique a son importance propre dans cette pensée «maudite» mais, aussi, pleinement «moderne». C'est cette dernière caractéristique, avec sa tragédie intérieure, qui peut comporter encore des éléments d'intérêt. 